\title{
The Variation of Absorption Edges of X-Rays for Liquid Hg-Rb Alloys
}

\author{
Toshio Itami*1 and Akitoshi Mizuno*2 \\ Division of Chemistry, Graduate School of Science, Hokkaido University, Sapporo 060-0810, Japan
}

The $\mathrm{L}_{\mathrm{I}}$ and $\mathrm{L}_{\mathrm{III}}$ edges of $\mathrm{Hg}$ and the $\mathrm{K}$ edge of $\mathrm{Rb}$ in liquid $\mathrm{Hg}-\mathrm{Rb}$ alloys were measured by the absorption spectroscopy of $\mathrm{X}$-rays. On increasing the $\mathrm{Rb}$ concentration, the absorption edges of $\mathrm{Hg}$ show an opposite behavior each other, the decrease of $\mathrm{L}_{\mathrm{I}}$ and the increase of $\mathrm{L}_{\mathrm{III}}$ compared with the case of pure liquid $\mathrm{Hg}$. This difference was discussed from the polyanion formation of $\mathrm{Hg}$ atoms on alloying. With the increase of alkali concentration, the $\mathrm{p}$ like state or polyanion seems to be situated on the lower energy side than that of $\mathrm{s}$ band. The electronic structure of this polyanion was discussed based on a simple LCAO analysis. Such an existence of polyanions may be responsible to the curious phenomena of liquid $\mathrm{Hg}$-alkali alloys, the maximum of the electrical resistivity at 60 at $\%$ alkali and the positively enhanced tendency in the intermediate alkali concentration range of the magnetic susceptibility in the overall negative deviation.

[doi:10.2320/matertrans.MAW200827]

(Received April 23, 2008; Accepted August 1, 2008; Published September 18, 2008)

Keywords: liquid metals, mercury, alkali, the absorption edge, compound formation

\section{Introduction}

In liquid alkali-polyvalent metal alloys, there exists a compound formation at some particular composition even in liquid alloys. ${ }^{1,2)}$ Such a typical system is liquid $\mathrm{Pb}-\mathrm{K}$ alloy. In this system, at the composition of 50 at $\% \mathrm{~K}$, the drastic increase of electrical resistivity occurs. This has been attributed to the formation of tetrahedral $\mathrm{Pb}_{4}{ }^{4-}$ polyanion, whose chemical bond is considered to be a bonding orbit derived from the overlap of p orbits. ${ }^{1-4)}$ The existence of this polyanion has been confirmed by the presence of a huge "prepeak" in the wave number range just before the first peak of the structure factor. Liquid mercury alloys, particularly liquid mercury-alkali alloys show also various curious phenomena, such as the minimum in the thermoelectric power, $Q$, at a few at $\%$ solute ("minimum in $Q$ " (see Fig. 1) ${ }^{5)}$ the maximum of electrical resistivity at 60 at $\%$ alkali, ${ }^{6}$ and the enhanced tendency in the intermediate concentration range of the magnetic susceptibility in the overall negative deviation tendency ("W shape" (see Fig. 2)). ${ }^{7-9)}$ The "minimum in $Q$ " appears for liquid alloys containing In, Tl, Li, Na, K, Rb, and $\mathrm{Cs}$ as a solute. Their phase diagrams ${ }^{10)}$ indicate many intermetallic compounds in the concentration range over 20 at $\%$ solute and there are no particular features around $\mathrm{Hg}$-rich region of a few at $\%$ solute, at which the "minimum in $Q$ " appears. There are no indications to resolve the cause of its origin. The "minimum in $Q$ " was described as a "mysterious phenomena" in the previous textbook (Faber 1972). ${ }^{11)}$

Up to date, as for the "minimum in $Q$ ", following points have been clarified by the author's group.

(1) "The minimum in $Q$ " appears in the mercury systems for which the heat of mixing is negative. ${ }^{5)}$

(2) It appears for the systems in which the electronegativity (or work function) difference between components is large. ${ }^{5)}$

\footnotetext{
${ }^{* 1}$ Present address: Japan Aerospace Explosion Agency (JAXA), 2-1-1 Sengen, Tsukuba 305-8505, Japan, Corresponding author, E-mail: itami@sci.hokudai.ac.jp

${ }^{*}$ Present address: Department of Physics, Faculty of Science, Gakushuin University, Tokyo 171-8588, Japan
}



Fig. 1 The concentration dependence of the thermoelectric power, $Q$, for liquid $\mathrm{Hg}$-alkali alloys.

(3) It is accompanied by the anomalous behavior of the temperature (or pressure) coefficient of physical quantities, such as the temperature coefficient of the electrical resistivity, thermoelectric power, magnetic susceptibility, atomic volume, and viscosity, partial molar entropy, and the pressure coefficient of thermoelectric power. ${ }^{7,12,13)}$

The points, (1) and (2), indicate that the "minimum in $Q$ " is caused by the charge transfer effect from solute (alkali) atoms to $\mathrm{Hg}$ ones. As is well known, the temperature coefficient and the pressure coefficient of the pair distribution function involve the three- and four-body distribution functions. Therefore the last point, (3), indicates that some local structure may appear due to this charge transfer effect. We presented the speculation that the "solvation structure" exists at a few at $\%$ solute in liquid $\mathrm{Hg}$ alloys for which the "minimum in $Q$ " can be seen. In this concentration range, positively charged alkali atoms may be surrounded with the partially charged $\mathrm{Hg}$ ones of negative sign. This situation is similar to the electrolyte water solution, in which the 


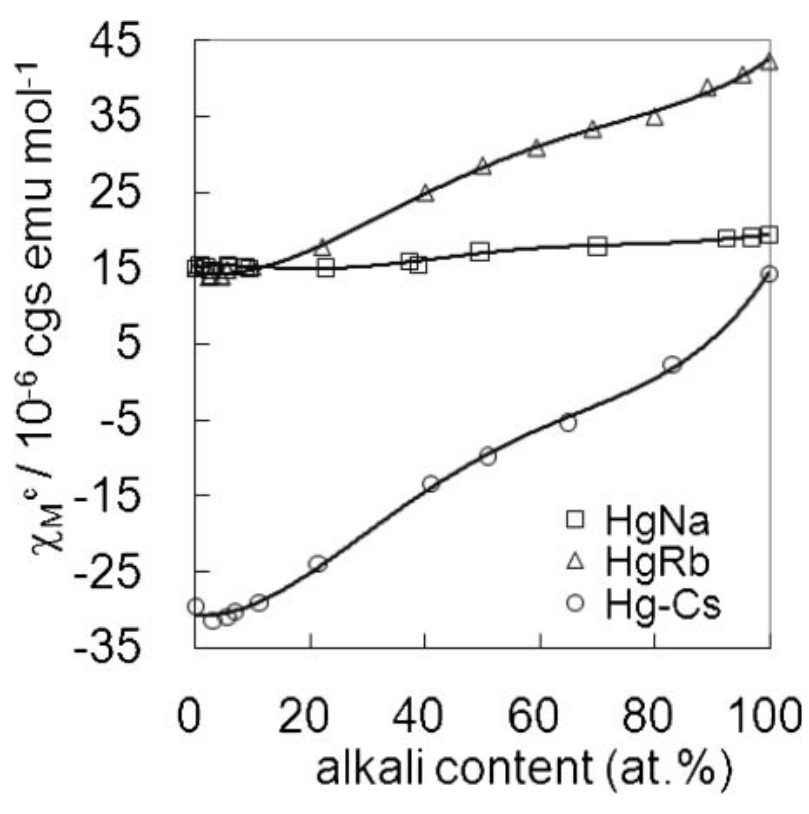

Fig. 2 The concentration dependence of the magnetic susceptibility, $\chi_{\mathrm{M}}^{\mathrm{c}}$, for liquid $\mathrm{Hg}-\mathrm{Na}, \mathrm{Hg}-\mathrm{Rb}$, and $\mathrm{Hg}-\mathrm{Cs}$ alloys. For liquid $\mathrm{Hg}-\mathrm{Cs}$ alloys, the total magnetic susceptibility, which is derived from contribution of the conduction electros (electronic part) and the ion core, is depicted and, for liquid $\mathrm{Hg}-\mathrm{Na}$ and $\mathrm{Hg}-\mathrm{Rb}$ alloys, only the contribution of electronic part is shown.

cation of, for example, $\mathrm{Na}^{+}$ion is surrounded with the oxygen atoms of water molecules, in which oxygen atoms possess a partially negative charge due to the polarization effect. $^{14)}$

Thus, the charge transfer effect is essential for the understanding of the physical properties of liquid $\mathrm{Hg}$-alkali alloys. This charge transfer effect may be also responsible to anomalous behaviors in the intermediate alkali concentration range, the maximum electrical resistivity at 60 at $\%$ alkali in common among all liquid Hg-alkali alloys, and the $\mathrm{W}$ shape of the magnetic susceptibility. In the previous paper, ${ }^{7)}$ a polyanion formation, such as $\mathrm{Hg}_{4}$ unit, was speculated around 60 at $\%$ alkali. Up to date, such a polyanion formation in liquid $\mathrm{Hg}$-alkali alloys has been discussed based on the analysis of EXAFS signal ${ }^{15}$ ) by comparing the analyzed interatomic distance of $\mathrm{Hg}-\mathrm{Hg}$ pair with that in the solid state obtained by the X-ray diffraction. It was also confirmed by the existence of the "prepeak" in the structure factor obtained by the neutron diffraction. ${ }^{16)}$ The Reverse Monte Carlo (RMC) analysis ${ }^{16)}$ of this structure factor also supports the existence of such polyanion. In this paper, such a polyanion formation is discussed from the X-ray absorption edge obtained in the European Synchrotron Radiation Facility (ESRF). Furthermore, this polyanion formation is supplemented by the discussion based on the linear combination of atomic orbitals (LCAO) method.

\section{Experimental}

The X-ray absorption spectra were measured for liquid $\mathrm{Hg}$ $\mathrm{Rb}$ alloys at the BM29 beamline in the ESRF. The energy range of synchrotron $\mathrm{X}$-ray was from the energy below the absorption edge of $\mathrm{Hg} \mathrm{L}_{\mathrm{I}}(14.839 \mathrm{keV}), \mathrm{Hg} \mathrm{L}_{\mathrm{III}}(12.284 \mathrm{keV})$

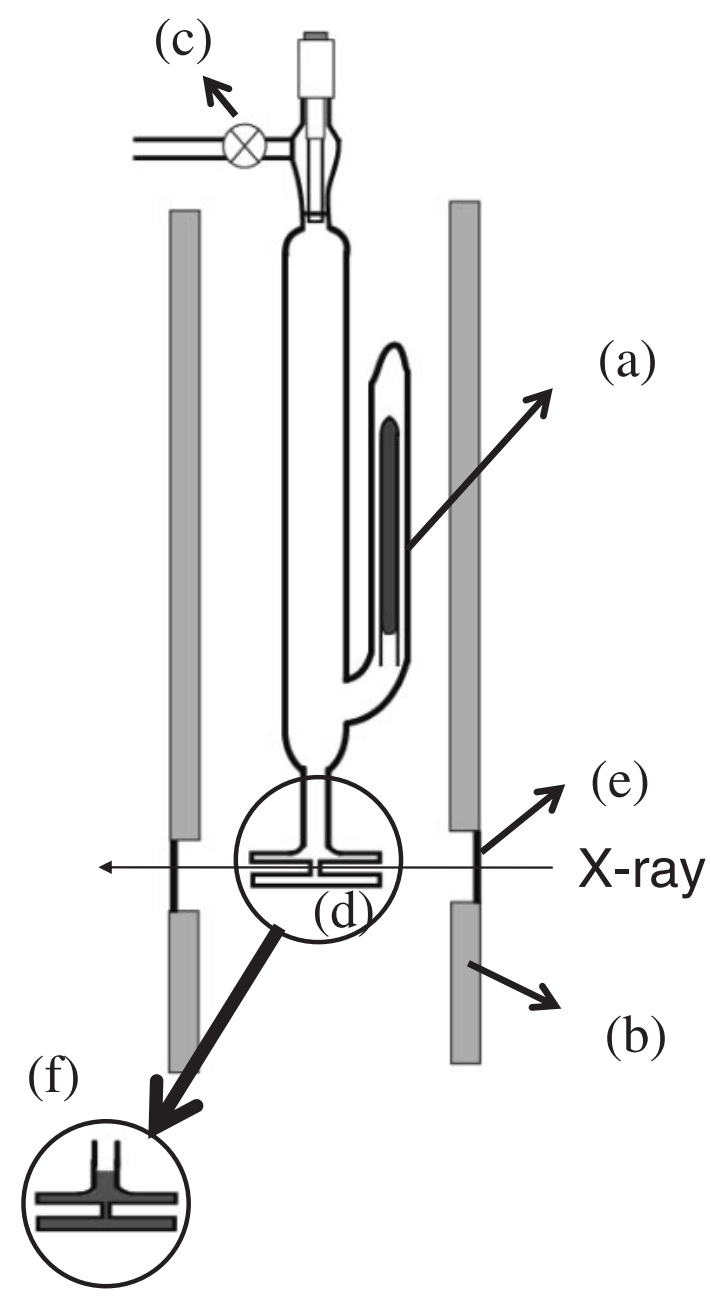

Fig. 3 The assembly of glass (quartz) cell; (a): sample ampoule port; (b): furnace; (c): cock for Ar gas inlet; (d): glass (quartz) cell part; (e): window (aluminum) for the X-ray; (f): glass (quartz) cell part containing liquid $\mathrm{Hg}-\mathrm{Rb}$ sample.

and $\mathrm{Rb} \mathrm{K}(15.200 \mathrm{keV})^{17)}$ to $15.6 \mathrm{keV}$. In the preliminary stage of experiment, the full cross-section of rectangular shape was confirmed for the transmitted X-ray beam which passed through the window (4 mm diameter) of glass (quartz) cell in Fig. 3. The X-ray absorption edge was determined by the inflection point of the transmitted X-ray intensity.

Strictly speaking, measured spectra contain the contribution of glass cell. However, in the preliminary X-ray absorption experiment, the spectrum of the blank cell shows a monotonous decrease with a small absorption. Probably this may be explained by the fact that the absorption $\mathrm{K}$ edges of $\mathrm{Si}$ $(1.838 \mathrm{keV})$ and $\mathrm{O}(0.531 \mathrm{keV})^{17)}$ of glass (quartz) cell were far distant from the energy range studied here. The effect of absorption of glass cell seems to be not so serious for the present study which is mainly concerned with the behavior of absorption edges for liquid $\mathrm{Hg}-\mathrm{Rb}$ alloys.

The alloy sample was prepared in an Ar-circulating glove box by weighing and introduced into a glass ampoule, which, in the next stage, was contained in a glass cell whose optical path has a size of $20-150 \mu \mathrm{m}$ length and $4 \mathrm{~mm}$ diameter. The glass ampoule containing the sample in the solid state was destroyed in the glass cell under a vacuum by adding the breaking force with the use of the screw. Then, the sample 
(a)

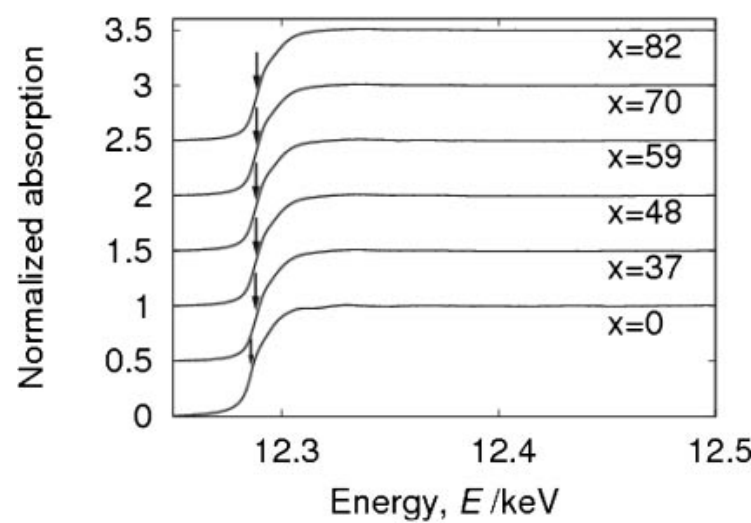

(b)

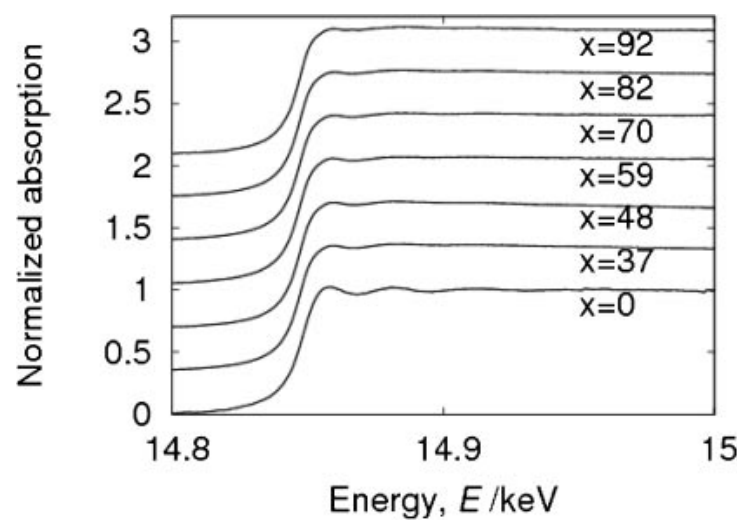

(c)

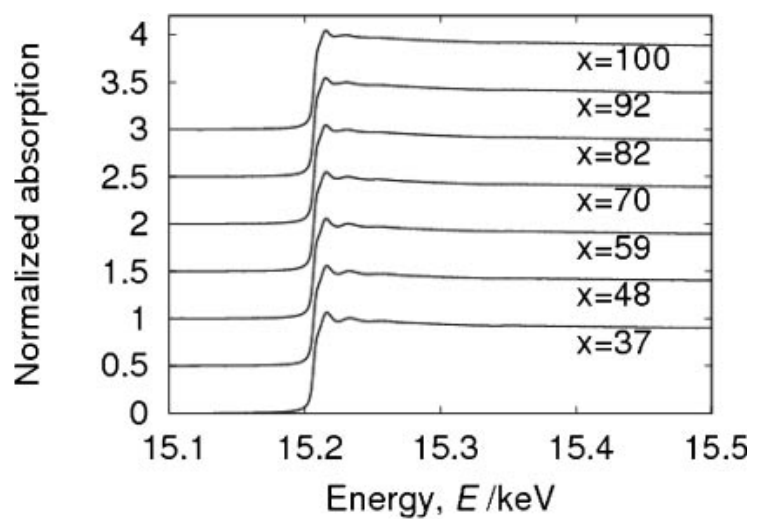

Fig. 4 Concentration dependence of the normalized X-ray absorption spectra of liquid $\mathrm{Hg}-\mathrm{Rb}$ alloys; (a): $\mathrm{Hg} \mathrm{L}_{\mathrm{III}}$ edge; (b): $\mathrm{Hg} \mathrm{L}_{\mathrm{I}}$ edge; (c): $\mathrm{Rb}$ K-edges. Positions of the absorption edges were indicated by arrows only in Fig. 4(a)

was melted and sufficiently homogenized in the liquid state. Finally, the liquid sample was introduced into the optical path region by Ar pressure. Figure 3 shows the assembly of the glass (quartz) cell, in which the broken glass ampoule containing a solid sample is schematically depicted. The experimental temperature was $50 \mathrm{~K}$ higher than the melting point.

\section{Results}

The obtained absorption spectra are shown in Fig. 4. These spectra show interesting behaviors on alloying, the decrease of the absorption edge for $\mathrm{Hg} \mathrm{L}_{\mathrm{I}}$ and $\mathrm{Rb} \mathrm{K}$ and the increase of

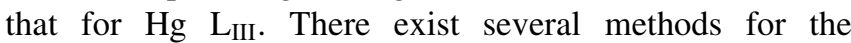

determination of the absorption edge. ${ }^{18,19)}$ Following to Lee et al. ${ }^{19)}$ the inflection point was adopted as the absorption edge in this study, as shown by arrows in the $\mathrm{L}_{\text {III }}$ edge in Fig. 4(a). Similar tendencies could be observed for the variation of the absorption edge on alloying even if it was determined by the energy of initial increase or that of the first maximum on the curves of absorption versus energy in Fig. $4{ }^{18)}$

The change of the absorption edge is very small compared with the energy of the incident X-ray. We noted the energy shifts of the absorption edges of liquid $\mathrm{Hg}-\mathrm{Rb}$ alloys compared with those of pure liquid $\mathrm{Hg}$ and $\mathrm{Rb}$, as shown in Table 1. Obtained energy shifts in the concentration range of $35-92$ at $\% \mathrm{Rb}$ were expressed as follows:

$$
\begin{array}{ll}
\Delta E_{0}=0.00797 x-0.698, & \text { for } \mathrm{Rb} K \text { edge; } \\
\Delta E_{0}=0.00188 x-1.403, & \text { for } \mathrm{Hg} \mathrm{L}_{\mathrm{I}} \text { edge; } \\
\Delta E_{0}=0.0114 x+1.574, & \text { for } \mathrm{Hg} \mathrm{L}_{\mathrm{III}} \text { edge. }
\end{array}
$$

In these equations, $x$ is the concentration of $\mathrm{Rb}$ in at $\%$.

\section{Discussions}

As can be seen in Table 1, it is very interesting that on alloying the $\mathrm{Hg} \mathrm{L} \mathrm{L}_{\mathrm{I}}$ edge decreases and the $\mathrm{Hg} \mathrm{L}_{\mathrm{III}}$ one increases. So far as authors know, such interesting behaviors of the absorption edge have been rarely reported for alloys in the solid state and the liquid state. The selection rule of this electron transition process is $\Delta l= \pm 1,{ }^{20)}$ which means that the difference of azimuthal quantum number, $l$, of electrons should be \pm 1 between the initial state and the final one. The $\mathrm{L}_{\mathrm{I}}$ edge corresponds to the electron transition from the initial state of $2 \mathrm{~s}$ inner core state to the final one of $\mathrm{p}$ type and the $\mathrm{L}_{\text {III }}$ edge to that from the initial state of $2 p$ core state to the final one of s type. In principle, the absorption edge should depend on the energy of both the initial state and the final one. However, for the absorption edges obtained in the present study, the final state around conduction band may be more deeply influenced on alloying compared with the initial one of deep core $2 \mathrm{~s}$ and $2 \mathrm{p}$ states. Thus, the change of the

Table 1 The concentration dependence of the energy shifts of the X-ray absorption edges in liquid $\mathrm{Hg}-\mathrm{Rb}$ alloys compared with those in pure liquid $\mathrm{Hg}$ and $\mathrm{Rb}$.

\begin{tabular}{cccc}
\hline $\mathrm{Rb}$ at\% & & $\Delta E_{0}(\mathrm{eV})$ & \\
\hline 0 & $\mathrm{Rb} K$ & $\mathrm{Hg} \mathrm{\textrm {L } _ { \mathrm { I } }}$ & $\mathrm{Hg} \mathrm{L}_{\mathrm{III}}$ \\
35 & & 0 & 0 \\
37 & -0.53 & -1.64 & 1.82 \\
48 & -0.42 & -1.23 & 2.06 \\
48 & -0.25 & -1.17 & 2 \\
53 & -0.26 & -1.27 & 2.29 \\
57 & -0.21 & -1.11 & 2.18 \\
59 & -0.17 & -1.23 & 2.39 \\
70 & -0.41 & -1.39 & 2.13 \\
75 & -0.06 & -1.23 & 2.42 \\
82 & -0.18 & -1.48 & 2.41 \\
92 & 0.12 & -1.29 & 2.56 \\
100 & 0.02 & -1.16 & 2.54 \\
\hline
\end{tabular}


absorption edge of $\mathrm{Hg}$ on alloying indicates that with the addition of alkali atoms the $\mathrm{p}$ like state becomes stable and the s like one unstable. This reasoning seems to be not inconsistent with the experimental fact, the decrease of $\mathrm{Rb}$ $\mathrm{K}$ edge (transition from initial $1 \mathrm{~s}$ state to final $\mathrm{p}$ type one) on alloying, shown in Table 1.

With the addition of alkali atoms into liquid $\mathrm{Hg}$, the distance between $\mathrm{Hg}$ atoms may increase. It is probable that the positively charged monovalent alkali ions do not contribute to the structure of energy band but only increase the sample volume or $\mathrm{Hg}-\mathrm{Hg}$ distance. The increase of the energy of the final s like state or s band on alloying may be understood by the increase of the $\mathrm{Hg}-\mathrm{Hg}$ distance, though this discussion depends slightly on the atomic distance dependence of band shape. However, it is very difficult to understand the decrease of the energy of the final $p$ like state on alloying, if this $\mathrm{p}$ like state is derived from the $6 \mathrm{p}$ band. Usually the $6 \mathrm{p}$ band is situated on the higher energy side compared with the 6s band even if they overlap each other. Authors consider that this stabilization of final $p$ like state is caused by the formation of atomic group of $\mathrm{Hg}$ atoms, polyanion, whose existence was confirmed as $\mathrm{Hg}_{4}$ planar unit in the solid $\mathrm{Hg}_{4} \mathrm{Na}_{6}$ compound ${ }^{21)}$ and $\mathrm{Hg}_{\mathrm{n}}(n=4$ or 5$)$ unit in the solid $\mathrm{Rb}_{5} \mathrm{Hg}_{19}{ }^{22)}$ by the $\mathrm{X}$-ray diffraction analysis. For liquid $\mathrm{Hg}$ alkali alloys, we stress the appearance of "inverse separation" of $6 \mathrm{~s}$ band and $6 \mathrm{p}$ state of $\mathrm{Hg}_{4}{ }^{6-}$ polyanion. The energy of $6 \mathrm{p}$ state of $\mathrm{Hg}_{4}{ }^{6-}$ polyanion is inversely in the lower energy side than that of $6 \mathrm{~s}$ band.

Here we note the existence of common composition of alkali concentration, 60 at $\%$ alkali $\left(\mathrm{Hg}_{4}(\mathrm{Alkali})_{6}\right)$, at which the maximum of the electrical resistivity are observed on its isotherms for liquid $\mathrm{Hg}$-alkali alloys. It is plausible to consider that the polyanion of $\mathrm{Hg}_{4}{ }^{6-}$ type may be responsible to this maximum of the electrical resistivity. Probably this polyanion is formed by only one part of $\mathrm{Hg}$ atoms among all $\mathrm{Hg}$ ones. In addition, the electrical conduction in liquid $\mathrm{Hg}$ alkali alloys is still carried out by the existence of vacant energy state in the overlapped sp band. The maximum of the electrical resistivity at 60 at\% alkali may be caused by the reason that the polyanion of $\mathrm{Hg}_{4}{ }^{6-}$ works as the strong scattering center against conduction electrons and its formation decreases the number of conduction electrons in the overlapped sp band.

As already described, the charge transfer from alkali atoms to $\mathrm{Hg}$ ones is the most fundamental factor to understand the curious physical properties of Hg-alkali alloys. With the proceed of charge transfer along with the increase of alkali concentration, the structure feature of liquid $\mathrm{Hg}$-alkali alloys changes from the "solvation" structure to the "polyanion" one, $\mathrm{Hg}_{4}{ }^{6-}$. There are two possibilities for the structure of $\mathrm{Hg}_{4}{ }^{6-}$ unit, planar square and tetrahedron. For the planar square array of $p_{z}$ orbits, a simple LCAO analysis provides one $p \pi$ bonding orbit, one $p \pi$ anti bonding one, and two $p \pi$ non-bonding orbits. ${ }^{23)}$ The bonding orbits of the planar square array are four $p \sigma$ orbits and one $p \pi$ orbit. $\mathrm{The}^{\mathrm{Hg}_{4}}{ }^{6-}$ unit has six p electrons which are transferred from alkali atoms. The occupied ratio of their bonding orbits is 0.6 . In the case of tetrahedral configuration, there are six $p \sigma$ orbits, ${ }^{23)}$ whose occupied ratio is 0.5 . Therefore, the planar square configuration seems to be more stable than the tetrahedral one, though, strictly speaking, the energy must be discussed. In addition, the existence of polyanion seems to be supported by the W shape of the magnetic susceptibility (Fig. 2), because the partial occupation of bonding orbits may enhance the paramagnetism. The existence of the $\mathrm{Hg}_{4}$ units in liquid $\mathrm{Hg}$-alkali alloys has been quite recently speculated in the quantum mechanical computational study, ${ }^{24)}$ though the calculation has been performed not for the condensed phase but for the Hg-alkali diatomics.

In the present study the polyanion formation was confirmed from the behaviors of X-ray absorption edges. The oscillation of absorption spectra also provides important information for such polyanion formation. In fact, it has been reported $^{15,16)}$ that the $\mathrm{Hg}-\mathrm{Hg}$ distance, $295 \mathrm{pm}$, obtained from the analysis of EXAFS oscillation for liquid $\mathrm{Hg}-\mathrm{Rb}$ alloys is in good accordance with that, $292 \mathrm{pm}$, given by the X-ray diffraction analysis for solid $\mathrm{Rb}_{5} \mathrm{Hg}_{19},{ }^{22)}$ for which $\mathrm{Hg}_{\mathrm{n}}$ unit has been reported. Thus, in the intermediate alkali concentration range in liquid $\mathrm{Hg}$-alkali alloys, the polyanion formation was confirmed also from the EXAFS study. Though the most advanced experimental tools, EXAFS, neutron scattering and the RMC were quoted for the present discussion, the discussion remains to be not so clear. This is caused by the moderate polyanion formation in the present liquid Hg-alkali alloys and the limitation of probabilistic nature of discussions for liquid state. The concentration of polyanion should be determined to make the present conclusion clear. For this purpose, the temperature dependence of NMR Knight shift and the heat of mixing are expected to be studied for liquid alkali alloys together with their theoretical analysis.

\section{Conclusions}

The X-ray absorption edge for liquid $\mathrm{Hg}-\mathrm{Rb}$ alloys provided some evidence of polyanion formation, which is responsible to the curious physical properties of liquid $\mathrm{Hg}$ alkali alloys in the intermediate alkali concentration range.

\section{Acknowledgement}

We are grateful to Prof. J. F. Jal, Prof. A. San-Miguel, and Dr. Felat in the University Claude Bernnard Lyon I and the European Synchrotron Radiation Facility for giving us the accommodation and the experimental chance.

\section{REFERENCES}

1) W. van der Lugt and W. Geetsma: Can. J. Phys. 63 (1987) 326-339.

2) W. van der Lugt: J. Phys. Condens. Matter 8 (1996) 6115-6125.

3) C. A. Coulson: Valence 2nd Ed. (Oxford University Press, 1961).

4) R. M. Hart, M. B. Robin and N. A. Kuebler: J. Chem. Phys. 42 (1965) 3631-3638.

5) T. Itami, S. Takahashi and M. Shimoji: J. Phys. F 14 (1984) 427-435

6) A. Mizuno, T. Itami, A. San-Miguel, G. Ferlat, J. F. Jal and M. Borowski: J. Non Cryst. Solids 312-314 (2002) 74-79.

7) T. Itami, K. Sugimura and Y. Yasuhara: J. Non Cryst. Solids 205-207 (1996) 455-458.

8) T. Itami, T. Sato and M. Shimoji: J. Phys. Soc. Jpn. 55 (1986) $2823-$ 2829.

9) T. Itami, K. Shimokawa, T. Sato and M. Shimoji: J. Phys. Soc. Jpn. 55 (1986) 3545-3551. 
10) ASM International Binary Phase Diagram 2nd Ed., The Materials Information Society, Material Park, OH (CD-ROM).

11) T. E. Faber: Introduction to the Theory of Liquid Metals, (Cambridge University Press, Cambridge, 1972).

12) T. Itami, T. Wada and M. Shimoji: J. Phys. F 12 (1982) 1959-1970.

13) M. Shimoji, T. Itami and Y. Morikawa: Zeit. für Physik. 156 (1988) 557-561.

14) P. Atkins and J. de Paula: Physical Chemistry 7th Ed., (Oxford University Press, 2002).

15) A. San-Miguel, G. Ferlat, J. F. Jal, A. Mizuno, T. Itami and M. Borowski: Phys. Rev. 65 (2002) 144203.1-4.

16) A. Mizuno, T. Itami, G. Ferlat, A. San-Miguel and J. F. Jal: J. Non Cryst. Solids 353 (2007) 3022-3026.
17) J. A. Bearden and A. F. Burr: Rev. Mod. Phys. 39 (1967) 125-141.

18) T. Oota (ed.): X-ray absorption spectroscopy-EXAFA and its Applications -, (ICP press. 2002, ISBN4-901493-21-3 C3054).

19) P. A. Lee, P. H. Citrin, P. Eisenberger and B. M. Kincaid: Rev. Modern Phys. 53 (1981) 769-806.

20) T. Ishii: Principles of The Theory of EXAFS, (Shokabo, Tokyo, 1994).

21) W. Nielsen and N. C. Baenziger: Acta Crystallogr. 7 (1954) 277282.

22) E. Biehl and H. J. Deiseroth: Z. Anorg. Allg. Chem. 625 (1999) 389-394.

23) W. L. Jolly: The Principle of Inorganic Chemistry, (McGraw-Hill, 1976).

24) E. Kraka and D. Cremer: Int. J. Mol. Sci. 9 (2008) 926-942. 